folk/ed. Derg, 2020; 26(1): 211-221

DOI: $10.22559 /$ folklor.1012

\title{
Roxelane Imaginée : Étude Sur L'ímage Contemporaine D'une Sultane
}

Roxelane as a Figment of the Imagination:

An Analysis of the Contemporary Imagery of a Sultana

\section{Özlem Kasap*}

\begin{abstract}
As important figures of the Ottoman history since the $16^{\text {th }}$ century Suleiman the Magnificent and his wife Roxelane, have become fundamental referents feeding societal imagery. This study focuses on the analysis of the 'imagery' of Roxelane; a woman who was able to hold power and attain a place in societal imagery in an age and setting where the role of women was extremely limited. A strong female archetype, a 'ruler' in an Empire where women did not rule and wield power, is created in the works studied through this imagery. The imagery associated with 'strong females' like Catherine de Médicis, Mary Tudor and Elizabeth Tudor who ruled their respective countries in the $16^{\text {th }}$ century, has gained a distinctive place in social memory. The imagery has found a wide audience, especially in its manifestation in fiction. The following study uses J.J. Wunenburger's definitions of imagery to examine the imagery of Roxelane and the ways in which it is recreated and re-used for differing functions in societal memory in two contemporary literary works.
\end{abstract}

Keywords: Roxelane, imagery, societal imagery, la magnifique, unofficial Hurrem

Geliş tarihi (Received):14 Mart 2019 - Kabul tarihi (Accepted): 05 Ocak 2020

Doç. Dr., Hacettepe Üniversitesi, Fransız Dili ve Edebiyatı Bölümü, Ankara, Türkiye. ozkasap@hacettepe.edu.tr. ORCID ID 0000-0003-4649-5689. 


\section{$\ddot{O} \mathbf{z}$}

\section{Hayal ürünü Roxelane: Bir kadın sultan imgesi üzerine inceleme}

Osmanlı İmparatorluğu'nun önemli tarihi kişilikleri içinde, Batılıların Muhteşem diye adlandırdıkları Kanunî Sultan Süleyman ve onun eşi Roxelane, Kanunî’nin hüküm sürdügü 16. yüzyıldan günümüze dek tiyatro yapıtı, roman, opera, bale türlerinde sayısız esere kaynaklık etmiş, bu yönüyle toplumsal imgelemi besleyen temel bir gönderge durumuna gelmiştir. Kanunî’nin ve Roxelane'ın yaşamından esinlenerek yaratılan ve beş yüz yıla yayılan yapıtlar aracılı̆̆ıyla tarihsel, toplumsal ve kültürel bir bakımdan Roxelane konusunda, toplumun güç konusunda ilgi odağını, algısını, tutumunu vb. yansıtan sayısız imge yaratılmıştır. Dolayısıyla bu çalışmada kadınların rollerinin alabildiğine kısıtlanmış olduğu bir uzamda, ortamda güç sahibi olmayı başarmış ve o güçle toplumsal imgelemde yer etmiş Roxelane “imgesi” üzerinde durulacaktır. Yapıtlarda gündeme gelen söz konusu imge arac1lığıyla, daha çok kadınların yönetimde olmadıkları bir imparatorlukta "yöneten" ve erk sahibi güçlü bir kadın arketipi yaratılmıştır. Söz konusu imge toplumsal olmakla kalmayıp evrenselleşmiş, 16. yüzyılda ülkelerini yönetmiş Catherine de Médicis, Mary Tudor ve Elizabeth Tudor gibi isimlere "güçlü kadın” imgeleriyle yakınlaştırılmış, toplumsal bellekte onlar gibi ayırıcı bir biçimde yer etmiştir. Yaratılan imge özellikle kurgusalın alanında dönüştürülerek geniş bir yer bulmuştur. Buna göre toplumsal bellekte yer etmiş Roxelane imgesinin Isaure de Saint Pierre'in La Magnifique (Muhteşem) (2004) romanında ve Özen Yula'nın Gayri Resmi Tarihe Göre Hurrem Sultan'dan Adap Erkân Dersleri veya Gayri Resmi Hurrem (1998) başlıklı tiyatro yapıtında hangi bakımlardan yeniden yaratıldığ 1 ve hangi işlevlerle yeniden kullanıldığı özellikle J.J. Wunenburger'in imge konusundaki tanımlamalarına koşut olarak incelenmektedir.

Anahtar sözcükler: Roxelane, Hurrem Sultan, imge, toplumsal imgelem, la magnifique

\section{Roxelane : De la figure à l'image}

Kanunî Sultan Süleyman, le législateur ou du nom que lui donne les Occidentaux, Soliman le Magnifique, dixième sultan de la dynastie ottomane est sans doute l'un des plus éminents monarques du XVI ${ }^{\text {ème }}$ siècle et son règne de 46 ans demeure le plus long de l'histoire de l'Empire ottomane. Soliman est certes une figure historique incontournable dont la représentation dans l'imaginaire populaire, à savoir la reproduction sous forme d'image(s) - que celle-ci évoque directement la réalité, qu'elle reflète une certaine facette de la réalité, ou encore qu'elle soit forgée sur une tout autre réalité et qu'elle devienne une représentation personnelle, une vision subjective, un nouveau produit d'imagination, donc de l'imaginaire populaire - ne surprend nullement. C'est un «personnage de légende ", " un monarque du Levant sorti tout droit d'une tragédie de Shakespeare » comme le présente le narrateur du documentaire $«$ Secrets d'Histoire - Soliman le Magnifique ${ }^{1}$. Toutefois, de cette figure exceptionnelle émane une seconde image qui se fraie une voie tout aussi glorieuse que celle 
du grand monarque, l'image d'une femme, d'une esclave : Roxelane. C'est justement à ce stade que l'étude de la création et de la réception d'une image intrigue, puisque l'intérêt porté à une femme, une figure féminine du monde ottoman au XVI ème siècle, vient égaler, voire dépasser celle du Magnifique. Que cette image de femme, apparue et glorifiée à une époque où son existence est rarement reliée directement au pouvoir, et surtout dans un espace où son rôle est fort limité, ouvre évidemment une voie où il est possible de traiter du processus que suit l'image de «femme » dans un contexte historique (oriental), chose qui s'avère délicate.

Ce présent travail se propose donc d'analyser une image reproduite à partir d'une figure historique très connue de l'histoire ottomane afin de suivre son évolution et de tenter de la redéfinir dans le nouveau contexte où elle est proposée et ensuite reçue dans la mémoire collective. La délicatesse de cette entreprise ne provient pas uniquement du rapport à la réalité du produit d'imagination mais des nombreuses qualifications qui sont ancrées dans les esprits. Qualifications qui établissent les contours de la réception puisque, sans les prendre en considération, il est difficile d'appréhender cette figure / image (pour ce qui est des personnages historiques qui ont été traités très longtemps et de nombreuses fois dans différents genres, il est possible de dire que la figure - le modèle - et l'image se confondent). L'image viendrait-elle même estomper la figure?

\section{La perception de l'image}

Jean-Jacques Wunenburger qui définit la perception de ce qui entoure une conscience, ici et maintenant, comme « le phénomène le plus immédiat » qui lui soit donné, précise que « la description philosophique de cette relation au monde fait déjà appel à la notion d'image, (...) une représentation sensible, concrète qui nous permet mentalement d'appréhender un donné extérieur à la conscience. » (Wunenburger, 2002: 15). L'image se présente donc comme un moyen de comprendre ce donné extérieur afin de l'intérioriser, d'en faire sien. Wunenburger voit dans cette représentation un aspect déconcertant puisque l'image " se situe à mi-chemin du concret et de l'abstrait, du réel et du pensé, du sensible et de l'intelligible » et qu'elle « permet de reproduire et d'intérioriser le monde, (...) de le faire varier, de le transformer jusqu'à en produire de fictifs » (Wunenburger, 1997: XI). Toutefois, il détermine, à partir des verbes imager, imaginaliser et imaginer, trois catégories différentes d'images qui indiquent trois intentionnalités différentes (Wunenburger, 2002: 23-24). Ces trois catégories permettraient de mieux distinguer les domaines de l'image et de mieux limiter celui qui constitue le nôtre. Imager mènerait au domaine de l'imagerie - « ensemble des images mentales et matérielles qui se présentent comme des reproductions du réel, en dépit des écarts et variations involontaires ou volontaires, par rapport au référent »-; imaginaliser à celui de l'imaginal - « qui renverrait plutôt à des représentations imagées que l'on pourrait nommer surréelles, (...) véritable plan originel des symboles [qui] actualise donc images euphoriques d'un sens qui nous dépasse et qui ne se laisse réduire ni à la reproduction, ni à la fiction »-et finalement imaginer, à l'imaginaire : « (...) l'imaginaire englobe les images qui se présentent comme des substitutions d'un réel absent, disparu ou inexistant, ouvrant ainsi un champ de représentation de l'irréel. Celui-ci peut se présenter comme (...) un jeu des possibles, comme dans le cas de la rêverie ou de la fiction (...)» (Wunenburger, 2002: 24)

Le support de cette étude étant deux œuvres de fiction, celles-ci puiseront donc leur 
inspiration dans ce troisième domaine où l'image est une substitution d'un réel qui n'est plus, d'un réel qui n'importe plus, l'image ayant devancé le réel. Aktulum précise que ce domaine fait référence à « la totalité des connaissances qu'une personne (...) ou qu'une société se fait / a reçu quant à une réalité générale » (Aktulum, 2014: 278). L'opération mentale qui rend possible la substitution ne pourrait se forger à l'insu de la totalité des connaissances qui reviendront forcément s'imposer suivant une certaine logique, associée à un mode d'expression adopté pour « convaincre ou persuader » car « Il ne suffit (...) pas de formuler des idées selon leur seule nécessité logique, mais il faut encore savoir les présenter de telle sorte qu'elles recueillent l'assentiment de ceux à qui elles sont destinées (...)» (Wunenburger, 2002: 28). De plus, ces images sont totalement soumises au « sujet interprétant qui est appelé à leur donner sens par des actes d'interprétations » (Wunenburger, 2002: 91).

Cet acte d'interprétation est certes l'acte par lequel l'auteur - le sujet interprétant - perçoit une réalité - historique dans ce cas - pour ensuite la rendre convaincante, captivante, à savoir plaisante si l'identification du lecteur est visée. Pour ce qui est de notre contexte historique et géographique, le puissant et terrifiant Orient du XVI ${ }^{\text {ème }}$ siècle, l'auteur doit considérer ses connaissances - qui, elles, sont déjà le produit d'une construction imprégnée d'idées toutes faites, qu'il s'agisse d'un auteur occidental se tournant vers l'Orient « pour trouver l'Orient qu'il veut trouver » (Ulağl1, 2007: 37) ou qu'il s'agisse d'un auteur oriental cherchant à interpréter plusieurs siècles plus tard ses propres terres, l'Orient de ses ancêtres - pour entreprendre la découverte de l'autre et par la suite le reconnaître. Le reconnaître étant essentiel pour la construction de l'image qu'il en fera mais aussi de sa propre image, de sa propre reconnaissance. Il paraît alors inévitable de rappeler les propos d'Edward Saïd qui précisait que « tout autant que l'Occident lui-même, l'Orient est une idée qui a une histoire et une tradition de pensée, une imagerie et un vocabulaire qui lui ont donné réalité et présence en Occident et pour l'Occident. Les deux entités géographiques se soutiennent ainsi et, dans une certaine mesure, se reflètent l'une l'autre. » (Saïd, 1980: 17) L'image de cet Orient reflète dans ce sens celle de l'Occident et vice-versa. Saïd rappelle dans la préface de son étude L'Orientalisme L'Orient créé par l'Occident que l'Orient est le « principal rival culturel » de l'Occident et qu'« il lui fournit l'une des images de l'Autre qui s'impriment le plus profondément en lui ». Un espace culturel ayant permis de définir l'Occident «par contraste : son idée, sa personnalité, son expérience » (Saïd, 1980: 14). Cependant, comme Saïd le souligne, cet Orient n'est pas « purement imaginaire ». Il s'inscrit « matériellement» dans l'espace occidentale, lui sert de support, le reflète, le complète, lui renvoie sa propre image, le définit.

Comment interpréter alors l'usage de l'image d'une femme, sa transformation à travers les siècles et les œuvres, sa présence au cœur d'histoires fictives portant chacune les traces de la vision du monde de leur auteur? Difficile de suivre le fil du processus de création de l'image de Roxelane, dès son origine jusqu'à la figure historique que cette femme fut. L'historien et orientaliste autrichien Joseph von Hammer relate, dans les notes et éclaircissements de son ample Histoire de l'Empire ottoman que Roxelane qui était désignée dans les rapports des ambassadeurs vénitiens et impériaux sous le nom de la Rossa (la Russe) serait « originaire de la petite Russie (la Galicie d'aujourd'hui), ou de la Lithuanie méridionale (l'Ukraine, la Volhynie et la Podolie) » et il ajoute ensuite que Roxelane pourrait en effet être « la fille d'un pauvre pope de Robatyn, petite ville située sur la Lipa, dans la Galicie. » (Hammer, 
1843: 487) Elle serait enlevée lors d'une razzia par des cosaques pour ensuite être vendue au Khan de Crimée qui l'offrira au harem de Soliman. Yermolenko, dans le recueil Roxolana in European Literature, History and Culture, affirme que ce que l'on connaît aujourd'hui au sujet de Roxelane n'est qu'interprétation et spéculation de la part de nombreux historiens, soi-disant historiens et dramaturges et que les premières rumeurs remontent au début des années 1530 où l'on apprit son mariage avec Soliman le Magnifique, événement qui surprit en Occident comme en Orient (Yermolenko, 2013: 51). Elle rappelle qu'à la seconde moitié du XVI ${ }^{\text {ème }}$ siècle, il y avait déjà des portraits de Roxelane, produits de l'imaginaire de l'Europe occidentale, et que Roxelane n'avait posé pour aucun de ces portraits.

La part du réel dans l'image de Roxelane ne serait donc que moindre, le réel étant mal/ peu/méconnu. Ce que nous croyons être le réel est déjà, même si pas totalement, recréé dans l'imaginaire populaire. L'histoire de cette esclave qui devînt Sultane se transforme assez vite, à partir d'une très brève information biographique, en une légende. Le fait que cette esclave « intelligente et ambitieuse » réussisse d'abord à se distinguer parmi quelque 300 femmes dans le harem impérial, à écarter la première compagne de Soliman, mère de son premier fils et à la renvoyer du harem, à éliminer Ibrahim Pacha, ami et grand vizir de Soliman qui se dresse comme un obstacle entre elle et le pouvoir, à faire de l'époux de sa fille, Rüstem, grand vizir, à comploter contre le prince prétendant au trône, Mustafa, jusqu'à le faire assassiner par son propre père et finalement à promettre au trône un de ses fils propose, à travers les siècles, de nombreux aspects prêts à être exploités. La multitude d'œuvres littéraires et musicales consacrées à cette femme hors du commun prouve combien son « image » se prête à diverses interprétations modelées suivants diverses intentions.

\section{Intention(s) d'auteur}

Les intentions d'auteur coïncideront forcément avec le style de celui-ci, la « beauté » de son texte. L'auteur écrit pour narrer une histoire, certes, mais pour la narrer à sa manière ; même si l'histoire a déjà été racontée, comme il en est maintes fois le cas dans celle de Roxelane; il se sent inspiré par cette histoire et veut raconter la « sienne ». Le but ultime n'est pas de reprendre l'histoire que nous venons de résumer quelques lignes plus haut, ou comme disait Alexandre Dumas, le «cadavre de l'histoire » tel qu'il est, mais plutôt de lui insuffler «la chaîne vitale $»^{2}$ qui donnerait le mouvement nécessaire et authentique à cette histoire.

Évidemment, ce mouvement a évolué à travers les siècles - n'oublions pas que cette image se réinvente depuis le XVI ${ }^{\text {İme }}$ siècle - et que les intentions d'auteur ne peuvent à elles seules expliquer cette évolution. Yermolenko précise que l'intérêt porté au meurtre de Mustafa reflétait parfaitement « la peur et l'admiration de l'Occident éprouvées envers l'Empire ottoman ». Cet événement nourrissait les idées toutes faites sur le Turc quant à la « cruauté », la « polygamie » et la " débauche ». Soliman avait tué son propre fils pour l'amour d'une femme, esclave de son extraordinaire harem ${ }^{3}$. Le rôle que Roxelane avait joué dans ce meurtre - on rapporte que ce sont ses complots qui avaient mené Soliman à douter de la loyauté de son fils, chéri par le peuple et surtout par les janissaires - évoquait la peur que semaient les puissantes femmes qui avaient marqué le XVI ème siècle : Catherine de Médicis, Mary Tudor et Elizabeth Tudor. Dans un contexte où les historiens et auteurs (le masculin des 
deux substantifs souligne particulièrement le sexe de ceux-ci) interrogeaient et critiquaient sérieusement le pouvoir et l'autorité de ces femmes, la part dévastatrice de Roxelane dans les affaires d'État de l'Empire ottoman démontrait à merveille combien le système patriarcal était menacé par les femmes (Yermolenko, 2013: 57).

Le pouvoir de Roxelane dans un monde oriental nécessitait une explication, la plus plausible semblait être la magie : l'historien britannique Richard Knolles la qualifiait comme la «maîtresse des pensées de Soliman » et l'accusait d'avoir corrompu, avec "sa beauté perfide, ses complots néfastes et sa magie noire » l'ordre politique de l'Empire et voyait en elle la raison de la régression de l'Empire. Sa constatation est loin d'être unique ; elle est reprise par d'autres ; et des mots comme sorcière, manipulatrice, calculatrice complètent souvent son nom ${ }^{4}$ (Yermolenko, 2013: 58). Cependant, l'Empire ottoman n'étant plus perçu comme une menace considérable suite à la défaite de la campagne de Vienne et surtout au traité de Karlowitz, un changement significatif s'opère dans la perception de l'Orient que cet empire constitue. Ce ne sont plus les campagnes militaires, les massacres, les horreurs de ce monde insolite qui hantent les esprits mais plutôt ses harems, et les amours exotiques qui s'y vivent (Yermolenko, 2013: 71-72). Avec cette nouvelle perception de l'Orient, l'image de Roxelane se fige en une image de femme ambitieuse et intelligente, sachant manipuler son entourage pour atteindre ses fins. Il ne s'agit plus d'une cruelle sorcière mais d'une figure de femme assez moderne qui s'élève dans un univers masculin. Yermolenko souligne qu'elle avait désormais sa place parmi les archétypes féminins comme Cléopâtre, Médée ou encore Lady Macbeth (Yermolenko, 2013: 81).

\section{La magnifique}

Le roman d'Isaure de Saint Pierre présente dans ce sens un exemple assez significatif puisqu'il obéit en quelque sorte à cette représentation moderne de Roxelane. Bien que le roman soit présenté comme « un conte oriental avec un peu d'eau de rose et pas mal de poison » dans le documentaire consacré à Roxelane et où l'auteur du roman intervient pour parler de son œuvre, ${ }^{5}$ et qu'il n'y aurait, d'après le présentateur Franc Ferrand, « endroit plus propice à tous les fantasmes » que le harem de Topkap1, la trame de l'histoire s'éloigne de la représentation traditionnelle des faits qui font l'histoire de Roxelane. Il va sans dire que cette histoire que nous avons essayé de résumer plus haut a servi des siècles durant à établir un certain message - qui a, comme nous venons de le préciser, évolué avec le temps - qui ne pouvait être que politique. Politique dans le sens où l'histoire a servi à dresser le portrait du Turc barbare tuant les siens et tous ceux qui s'opposent à son règne; le Turc cruel qui obligeait à la servilité des femmes arrachées à leur terre et à leur famille. Portrait d'un Oriental face à ses esclaves Occidentaux, 1'Islam face au Christianisme. ${ }^{6}$ Si ce n'est, bien sûr pour utiliser le harem comme décor à une simple histoire exotico-érotique. Il faudrait évidemment préciser que cette contextualisation simpliste n'est plus à la mode au $\mathrm{XX}^{\text {ème }}$ et $\mathrm{XXI}^{\text {ème }}$ siècles où les auteurs recherchent un angle authentique pour raconter ce 4 conte oriental ${ }^{7}$. La Magnifique se distingue par son approche méticuleuse - une remarquable documentation complète le roman- au personnage que l'auteure développe à travers les pages sans succomber à la tentation d'en faire une marionnette unidimensionnelle dont la présence ne s'explique que 
par d'ignobles machinations. Il semble bien que la curiosité d'Isaure de Saint Pierre vis-à-vis de l'histoire de Roxelane est plutôt aiguisée par son intelligence - et non dans la connotation défavorable de cet aspect - que Saint Pierre résume par ses mots : " elle avait une tête très politique $»^{8}$. Ses paroles rejoignent les propos de Kenizé Mourad prononcés dans le documentaire « Secrets d'histoire ». Mourad dit de Roxelane qu' « elle était d'une intelligence très grande et [qu'] elle avait le sens de l'intrigue ». Documentaire où on entend également le narrateur dire qu' : « Il [Soliman] aimera et respectera sa volonté farouche ${ }^{9}$ d'exister à l'égal des hommes même dans ce cadre étroit et particulier du harem. ${ }^{10}$ Ces mots rappellent tout de suite le merveilleux titre du journaliste du New York Times qui dans son article où il présente une œuvre sur Roxelane, clame : The Woman Who Smashed a Glass Ceiling in the 16th Century (La femme qui a a brisé un plafond de verre ${ }^{11}$ au XVI ${ }^{m e}$ siècle) (Madden 2017). Cette référence propre au $\mathrm{XX}^{\mathrm{ème}}$ siècle qui démontrerait que l'image de Roxelane continue son évolution dans l'imaginaire populaire où elle se retrouve modernisée, rendrait également explicite la voie qu'Isaure de Saint Pierre adopte pour construire sa Roxelane.

Effectivement, Roxelane, ainsi peinte par Saint Pierre, est un personnage ambigu errant du sentiment de captivité et de servilité à sa soif du pouvoir : " Qui savait quels rêves de grandeur et de puissance s'abritaient sous ce joli crâne ?» (Saint-Pierre, 2002: 71). L'inquiétude éveillée par les rêves de cette esclave paraît légitime puisque nous voyons se déployer les efforts d'une femme décidée à se construire un univers où elle pourrait vivre une « vraie vie » bien qu'enfermée dans une " prison dorée, prison élargie, mais prison tout de même » (Saint-Pierre, 2002: 103). L'originalité que Saint Pierre attribue à Roxelane est qu'elle la rend consciente de sa servilité tout en la rendant consciente que la liberté dont elle disposait avant d'être ravie ne pouvait égaler le pouvoir qui se présentait à elle. «Mais qu'était-ce la liberté dans un village sordide. » Alors que le harem offrait « une perspective inouïe pour la petite paysanne qu'elle avait été » (Saint-Pierre, 2002: 63). Il est clair que l'auteure attribue à cette jeune femme la clairvoyance nécessaire lui permettant d'être maîtresse de son sort : « l'Empire ottoman régnait sur Constantinople et sur le monde, et c'était son empereur qu'elle tenait entre ses bras, prodigieux destin pour la fille d'un pauvre pope » (Saint Pierre, 2002: 103). Isaure de Saint Pierre propose une image de femme prête à revendiquer ce qui devrait lui revenir, un pouvoir en proportion avec son intelligence, un pouvoir que sa féminité n'empêcherait d'exercer car ce n'était pas la servilité qui empêchait l'ascension dans l'Empire mais la condition de femme. C'est ainsi que l'auteure interprète la haine de Roxelane pour Ibrahim quand celui-ci est nommé grand vizir:

« - Comment pourrais-je lutter contre tant de grâce et de qualités viriles, moi qui ne suis que de la 'chair vendue'?

- Ibrahim l'est aussi, ne l'oublie pas.

- Oui, mais il n'est pas enfermé comme moi dans un sérail. Oh, je hais cet enfermement et je hais d'être femme » (Saint-Pierre, 2002: 110).

Nous pourrions dire que le personnage se charge d'un rôle difficile, celui d'une femme intelligente qui s'empare d'un pouvoir mérité. L'image de Roxelane, l'image que nous pensons connaître s'efface en quelque sorte dans la trame de l'histoire puisque le cadre peut changer, l'époque peut changer, les noms peuvent changer mais la volonté « farouche » de 
cette femme trouvera chez les lecteurs, notamment chez les femmes, de bons auditeurs.

«Que de chemin parcouru depuis qu'on l'avait enlevée à son petit village si pauvre! Si elle y était restée, elle serait aujourd'hui une vieille paysanne flétrie et illettrée, alors qu'elle était la maîtresse de l'Empire » (Saint-Pierre, 2002: 342).

Contemporain d'Isaure de Saint Pierre, Özen Yula, dramaturge turc, écrit en 1998 une pièce de théâtre dans laquelle il reprend cette même histoire pour mettre en scène un spectacle qui dévoilerait les étroits rapports existant entre la fiction et la réalité, à savoir entre « le récit et l'Histoire » (Yermolenko, 2013 : 213). Il s'agit effectivement d'une parodie critique du genre en question, roman ou théâtre historique. Dans cette pièce intitulée Gayri Resmi Tarihe Göre Hurrem Sultan'dan Adap Erkân Dersleri veya Gayri Resmi Hurrem (Cours de savoir-vivre proposés par Hurrem Sultan d'après l'Histoire non-officielle ou Hurrem nonofficielle $)^{12}$ Yula nous présente une Roxelane qui est appelée de son nom turc Hurrem Sultan déjà âgée, fatiguée des intrigues du harem, s'enfermant dans une chambre secrète et ayant comme seule compagne une jeune esclave qui semble être la jeune Hurrem. Usant de la technique du théâtre dans le théâtre ${ }^{13}$, qui rappelle fortement les pièces de Jean Genet où personne n'est celui qu'il semble être, Yula reflète une image de Roxelane / Hurrem qui diffère de l'image de Saint Pierre. Hurrem non seulement divague dans le temps - elle est tantôt dans le présent, tantôt dans le passé - mais aussi se perd dans diverses identités. Il n'y a pas une unique Hurrem (comme il n'y a plus une unique figure mais une multitude d'images forgées à partir d'elle). D'ailleurs, l'objectif de Yula, en mettant en scène une femme performer Hurrem Sultan, est de démystifier la machine qu'est la fiction tout en démontrant comment les diverses identités qui sont ainsi mises sur scène représentent la perte d'identité qu'impose finalement l'enfermement de ces femmes.

Dès la première scène, Yula démonte le processus de création du personnage (dans ce cas historique) et met en évidence le rapport établi entre la personne et le personnage.

«Hurrem : Je n'ai raconté mon passé à personne. C'est là où réside la source de mon pouvoir. Quand ils ne savent pas, ils inventent un passé à leur guise. La légende nourrit la légende. Ils ont dit que j'étais d'origine polonaise, puis, française, russe, italienne... Ils n'ont pas su, plus ils n'ont pas su, plus ils ont raconté, plus ils ont raconté, plus ils ont créé une toute autre Hurrem ${ }^{14} \gg$ (Yula, 2007: 13).

Ces paroles du protagoniste répondent parfaitement à la question qu'on pourrait se poser au sujet de la «femme » qui se dissimule derrière chaque représentation qui en est faite. Chaque fois qu'elle est racontée, elle s'éloigne encore plus de ce qu'elle a pu être. L'image qui se multiplie n'est jamais un reflet conforme à son modèle et n'est finalement que l'écorce servant à protéger l'histoire. « (...)tout ce qui est raconté se transforme en une nouvelle histoire. Il s'agit de l'histoire des personnes racontées, des événements mais aussi de l'histoire de celui qui la raconte $\gg^{15}$ (Yula, 2007: 24). Quant à la prise de position de Hurrem vis-à-vis du pouvoir et de sa vie telle qu'elle était avant son ravissement, il serait possible de dire que Yula s'engage dans une voie tout à fait opposée à celle de Saint Pierre, puisque dans ses divagations Hurrem parle des beautés d'une vie simple, comme dans son enfance. Elle regrette les petites choses qui embellissent la vie et rêve de refaire sa vie dans les rues de Robatyn : 
« Dans les rues de Robatyn (...) je vais me refaire un tout nouveau passé. Un passé loin du sang, de la haine, de l'ambition, des guerres et des accords. Je vais vieillir au bord d'une rivière en écoutant le bruit de l'eau. (...) Je le mérite... Je vais me réveiller chaque matin remplie de joie. Comme dans mon enfance. Ça fait si longtemps que je ne me réveille pas avec joie (...) ! Je me réveille sans cesse à des matins obscurs, cauchemardesques ${ }^{16}$ (Yula, 2007: 57 - 58).

Cependant, la dernière scène de cette pièce en deux actes révèle la réalité quant à l'identité de Hurrem et de la jeune esclave. Deux femmes jouaient leur rôle dans une pièce destinée aux femmes du harem de Handan Sultan, une trentaine d'années après la mort de Hurrem; et ainsi le quatrième mur était ainsi rendu visible.

\section{Conclusion}

Ces deux œuvres fictives de deux auteurs de nationalités et de sexe différents montrent combien l'image reproduite à partir d'une figure est malléable. Une fois introduite dans l'imaginaire populaire, l'image devient un instrument incontournable offrant une multitude de possibilités. Ainsi, l'image de femme brisant le plafond de verre dans le roman d'Isaure de Saint Pierre devient dans la pièce d'Özen Yula l'image de femme brisant le mystère de la fiction. En montrant le processus même de création et de représentation sur scène, en semant le doute sur ce qui est présenté, sur le réel, sur le transmis, Yula contribue d'une certaine façon à l'idée qu'une image est forcément polysémique et porteuse d'intention(s) explicite(s) comme implicite(s).

Dans ce cadre, le roman d'Isaure de Saint Pierre traduisant l'intention de faire de Roxelane « la » femme se hissant au sommet de la hiérarchie du pouvoir, il serait possible de préciser que l'image qu'elle reflète est celle de « la femme intelligente et ambitieuse » et que le reste - intrigues, meurtres, amours, exotisme - vient compléter le décor propice à la narration. C'est sans doute la raison pour laquelle la servilité et l'enfermement des femmes, qui apparaissent généralement comme des thèmes majeurs quand un auteur traite de l'histoire de Roxelane, ne sont pas mis en emphase alors que chez Yula, ces thèmes deviennent la trame même de l'histoire, d'autant plus qu'ils sont accentués par l'espace dans lequel se déroule la pièce : chambre secrète bien cloitrée où nul n'entre ni en sort. De par le choix de cet espace, une chambre close ménagée dans le harem (clos), Yula laisse assez bien deviner son intention : traiter de la crise d'identité de femmes enfermées dans un espace clos mais aussi enfermées dans les rôles qui leur sont attribués ; images au-delà desquelles l'on ne peut plus les reconnaître.

\section{Endnotes}

1 Secrets d'Histoire - Soliman le Magnifique, Août 2012.

2 « [...] après avoir étudié l'un après l'autre la chronique, l'histoire et le roman historique, après avoir bien reconnu que la chronique ne peut être considérée que comme source où l'on doit puiser, nous avons espéré qu'il restait une place à prendre entre ces hommes qui n'ont point assez d'imagination et ces hommes qui en ont trop ; nous nous sommes convaincus que les dates et les faits chronologiques ne manquaient d'intérêt que parce qu'aucune chaîne vitale ne les unissait entre eux, et que le cadavre de l'histoire ne nous paraissait si repoussant que parce que ceux qui l'avaient préparé avaient commencé par enlever les chairs nécessaires à la ressemblance, 
les muscles nécessaires au mouvement, enfin les organes nécessaires à la vie [...] » Cité par Gengembre, G. (2010)

3 Dans son étude, Le Harem et l'Occident, Fatema Mernissi rapporte que le sourire pouvait trahir les pensées et qu'il était possible de diviser la partie masculine de l'Occident en deux catégories: "Les Américains d'un côté, les Européens de l'autre. Les Américains, lorsqu'ils prononcent le mot 'harem', ont un sourire franchement et carrément embarrassé; quel que soit le sens qu'ils donnent à ce mot, il paraît évident qu'il évoque pour eux quelque chose qui est du ressort de la honte. Les Européens offrent dans leurs sourires une gamme de sentiments plus étendue, qui va de la gêne polie à l'exubérance la plus joviale. Ces nuances subtiles dépendent de la distance qui separe les uns et les autres des rives de la Méditéranée: au sud, Français, Espagnols et Italiens y mettent quelque chose d'aguicheur; au nord, Scandinaves et Allemands paraissent plutôt étonnés (...)." (Mernissi 2001: 17). Il s'avère certes difficile de tenter une étude d'image de Roxelane sans plonger dans la perception du "harem", espace qui explique la multitude d'interprétations, bien souvent enrobées dans l'exotisme oriental et pimentées d'un zest d'érotisme. Cependant, le harem comme espace particulier est le sujet d'un autre travail (en cours). Nous pourrions peut-être nous contenter de rappeler que bon nombre d'auteurs, dramaturges, peintres s'étaient servi de cet espace dépeint comme licencieux pour définir le despotisme oriental. (Lewis 2006: 146)

4 “On disait que c'était une sorcière et qu'elle avait fait absorber à Soliman des filtres, c'était ça l'explication de ce qui paraissait inexplicable. (...) L'historien n'a pas de documents positifs pour expliquer les choses mais (...) il y a une tendance dans la personnalité de Soliman à être comme ça sous la coupe de fortes personnalités.” Gilles Veinstein dans Secrets d'Histoire - Soliman le Magnifique, Août 2012.

5 L'intégrale - Roxelane, l'épouse adorée de Soliman le Magnifique, 14/02/2012

6 Kasap, Özlem. 2006. Soliman le Magnifique et ses proches: de l'Histoire à la fiction. (Thèse de doctorat non publiée)

7 Les romans L'aurore des bien-aimés et Grand-Seigneur de Louis Gardel peuvent être cités dans ce cadre.

8 L'intégrale - Roxelane, l'épouse adorée de Soliman le Magnifique, 14/02/2012

9 L'adjectif est souligné de notre part pour montrer qu'aujourd'hui même cette volonté ne paraît toujours pas ordinaire.

10 Secrets d'Histoire - Soliman le Magnifique, Août 2012.

11 Le plafond de verre est une expression inventée vers la fin des années 70 aux Etats-Unis par les sociologues féministes et désigne que les niveaux supérieurs ne sont pas accessibles aux femmes. Dans son discours après sa défaite face à Donald Trump en 2016, Hillary Clinton se plaignait de n'avoir pu briser ce plafond de verre. (Hulot-Guiot 2016).

12 Cette pièce de théâtre est écrite en 1998 et la première édition dans les œuvres complètes date de 2002, nous ferons référence au texte publié en 2007.

13 La pièce tourne autour d'un personnage principal qui nous est présenté comme Hurrem Sultan, et d'une jeune esclave qui la seconde. Celle-ci fait tantôt parler des marionnettes qui représentent la cour impériale, tantôt devient Hurrem Sultan alors que l'autre joue le rôle de l'esclave. A la fin, le lecteur / spectateur apprend que les deux femmes ne sont pas celles que nous croyions et qu'elles jouent en réalité (!) un rôle dans un spectacle mis en scène au harem pour Handan Sultan (compagne du sultan Mehmed III) et que la jeune fille n'est autre que Mahpeyker qui deviendra plus tard la fameuse Kösem Sultan, épouse du Sultan Ahmed I. Yula utilise donc une éminente figure féminine de l'histoire ottomane pour raconter celle d'une autre, tout aussi éminente.

14 Le texte de Yula est en turc, les traductions nous appartiennent. Hurrem : Geçmişimi kimseye anlatmadım. Gücümün kaynă̆ da bu zaten. Bilmedikleri zaman kendilerince bir geçmiş kurarlar. Efsane, efsaneyi besler. Polonya asıllı olduğumu söylediler, sonra... Fransız, Rus, Ittalyan asıllı olduğumu... Bilmediler, bilmedikçe anlattılar, anlattıkça bambaşka bir Hurrem yarattılar.

15 Her anlatılan, yeni yazılan bir hikâyeye dönüşür. Anlatılan kişilerin, olayların olduğu gibi, anlatıcının kendi hikâyesidir de bu.

16 Rotagina sokaklarında gezerken (...) yepyeni bir geçmiş yaratacağım kendime. Kandan, kinden, ihtirastan, savaşlardan ve antlaşmalardan uzak bir geçmiş olacak. Bir ırmağın kıyısında suyun sesini dinleyerek yaşlanacağım. (...) Bunu hak ediyorum... Sevinç dolu uyanacă̆ım sabahlara. Tıpkı çocukluğumdaki gibi. Ben sevinç dolu uyanmayalı o kadar uzun zaman oldu ki (...)! Karanlık, kabus dolu sabahlara kalkiyorum hep. 


\section{Bibliographie}

Aktulum, K. (2014). Folklorik imgelem. Bir toplumsal imgeler rezervuarı olarak imgelemin epistemolojik temellerine giriş (L'imaginaire folklorique, introduction aux fondements épistémologiques de l'imaginaire, en tant qu'un réservoir des images sociales). Milli Folklor, S. 101, ss. 277-290.

Kasap, Ö. (2006). Soliman le magnifique et ses proches : de l'histoire à la fiction. Thèse de doctorat non publiée.

Lewis, R. (2006). Oryantalizmi yeniden düşünmek - kadınlar, seyahat ve Osmanlı haremi (Rethinking Orientalism: Women, travel and the Ottoman harem) (B. Uygun - Aytemiz, Ş. Başlı, Çev.) İstanbul: Kap1.

Mernissi, F. (2001). Le harem et l'occident. Paris: Albin Michel.

Saï, E. (1980). L'orientalisme - l'orient créé par l'occident. Paris: Seuil.

Saint Pierre, I. (2004). La magnifique. Paris: Le Livre de Poche.

Ulağl1, S. (2007). L'image de l'orient turc dans la littérature française, les idées, les stéréotypes et les stratégies. Istanbul : Isis.

Wunenburger, J-J. (1997). La philosophie des images. Paris: Universitaires de France.

Wunenburger, J-J. (2002). La vie des images. Grenoble: Universitaires de Grenoble.

Yermolenko, G. İ. (2013). Avrupa edebiyatı, tarihi ve kültüründe Hurrem Sultan (Roxolana in European literature, history and culture). (F. B. Aydar, Çev.). Istanbul: Koç Üniversitesi.

Yula, Ö. (2007). Toplu oyunları 3. Istanbul: YKY.

\section{Références électroniques}

Gengembre, G. (2010). Le roman historique : Mensonge historique ou vérité romanesque ?. Études, tome 413(10), 367-377. Consulté le 18/10/2018 sur https://www.cairn.info/revue-etudes-2010-10page-367.htm.

L'intégrale - Roxelane, l'épouse adorée de Soliman le magnifique, 14/02/2012 (documentaire) www. europe1.fr.

Secrets d'histoire - soliman le magnifique, août 2012 (documentaire) https://gloria.tv/video/ rfN2nWVuAu4z4T19ReSnoUpSY .

Hammer-Purgstall, J. (1835-1843). Histoire de l'empire ottoman, depuis son origine jusqu'à nos jours. Consulté le 11/09/2018 sur gallica.bnf.fr.

Hullot-Guiot, K. (2016). Qu'est-ce que le 'plafond de verre'?. Libération

Consulté le 2/08/2018 sur http://www.liberation.fr/planete/2016/11/10/qu-est-ce-que-le-plafond-deverre 1527503 .

Madden, T. F. (2017). The Woman who smashed a glass ceiling in the 16th century. New York Times

Consulté le 2/08/2018 sur https://www.nytimes.com/2017/11/22/books/review/leslie-peirce-empressof-the-east-roxelana-hurrem-sultan.html. 